\title{
Inverted Solutions of KdV-Type and Gardner Equations
}

\author{
A. KARCZEWSKA ${ }^{a}$ AND P. RozMeJ ${ }^{b, *}$ \\ ${ }^{a}$ Institute of Mathematics, University of Zielona Góra, \\ Szafrana 4a, 65-246 Zielona Góra, Poland \\ ${ }^{b}$ Institute of Physics, University of Zielona Góra, \\ Szafrana 4a, 65-246 Zielona Góra, Poland
}

Received: 12.09.2021 \& Accepted: 08.11.2021

Doi: 10.12693/APhysPolA.140.445

*e-mail: p.rozmej@if.uz.zgora.pl

\begin{abstract}
In most of the studies concerning nonlinear wave equations of Korteweg-de Vries type, the authors focus on waves of elevation. Such waves have general form $u_{\mathrm{u}}(x, t)=A f(x-v t)$, where $A>0$. In this paper we show that if $u_{\mathrm{up}}(x, t)=A f(x-v t)$ is the solution of a given nonlinear equation, then $u_{\text {down }}(x, t)=-A f(x-v t)$, i.e. the inverted wave is the solution of the same equation, but with the changed sign of the parameter $\alpha$. This property is common for Korteweg-de Vries equation, extended Korteweg-de Vries equation, fifth-order Korteweg-de Vries equation, Gardner equation, and their generalizations for cases with an uneven bottom.
\end{abstract}

topics: solitons, KdV-type equations, Gardner equation, travelling wave solutions

\section{Introduction}

The first observations of soliton waves by Russel [1] and their interpretation by Boussinesq [2] led to the Korteweg-de Vries equation (KdV) [3] in the nineteenth century. Korteweg and de Vries have emphasized in [3] that solitary waves may involve both a positive (elevation) and a negative (depression) localized perturbation, depending on the sign of dispersion. Waves of elevation, mostly observed in seas and oceans, are naturally induced by phenomena such as strong wind gusts, ocean currents and tides. This is why most quantitative studies have reported the elevation solitary waves. In the case of waves on the surface of a fluid, elevation solitary waves can only be observed in the long wavelength limit when gravity is dominant. For thin fluid layers, when surface tension is no longer negligible, capillary effects have a drastic influence on extended waves and localized waves, such as KdV solitary waves, which are predicted to become the depression waves rather than elevation ones. Such depression solitary surface waves have been first observed on thin layers of mercury in [4]. Other known cases of depression solutions are given from the Gardner equation, in particular for internal waves $[5,6]$.

A growing number of observations and facts in the literature (primarily satellite images) indicate the presence of internal nonlinear waves near the shelves. Under specific conditions (depth, salinity, temperature), soliton giant depression waves with amplitude up to several tens of meters and length up to several hundreds of meters may form $[5,6]$.
Such waves can cause dramatic effects. The paper [6] shows that such waves of negative amplitude could be the most likely cause of several submarine disasters. These facts were the motivation to discuss the properties of solutions of nonlinear wave equations of $\mathrm{KdV}$-type corresponding to the depression waves.

The present study shows that four kinds of nonlinear wave equations, namely $\mathrm{KdV}$, extended $\mathrm{KdV}$, fifth-order $\mathrm{KdV}$, and Gardner equations, admit depression solutions for the negative sign of nonlinearity parameter $\alpha$. Moreover, the same property holds for all these equations, generalized for the uneven bottom.

\section{Flat bottom case}

Let us begin with the simplest cases when the bottom of the fluid container is flat. Since we will also consider the cases with an uneven bottom later, we use equations corresponding to the fixed reference frame. We will also show that all properties discussed by us in this paper that are valid in a fixed reference frame remain valid in a frame moving at a constant velocity.

\subsection{Korteweg-de Vries equation}

Korteweg-de Vries equation in the fixed frame has the following form (subscripts stand for partial derivatives)

$$
u_{t}+u_{x}+\frac{3}{2} \alpha u u_{x}+\frac{\beta}{6} u_{x x x}=0 .
$$


Recall that the KdV equation applies for long surface waves with small amplitude, because it was derived assuming that the two parameters

$$
\alpha=\frac{A}{H} \quad \text { and } \quad \beta=\left(\frac{H}{L}\right)^{2}
$$

are small and of the same order ( $A$ denotes the wave amplitude, $H$ - the basin depth, and $L$ the wavelength).

$\mathrm{KdV}$ equation (1) has analytic $n$-soliton solutions and two kinds of periodic solutions, cnoidal and superposition ones. Single soliton and periodic solutions have the form of "travelling waves", that is, the waves with a fixed profile, moving with a constant speed. Such waves are described by the following function

$$
u(x, t)=A f[B(x-v t)]+D .
$$

For a single soliton solution one has (denoting $\xi=$ $x-v t)$

$$
f(\xi)=\operatorname{sech}(B \xi)^{2},
$$

where

$$
\begin{aligned}
& B=\sqrt{\frac{3 \alpha}{4 \beta} A,} \\
& v=1+\frac{\alpha}{2} A,
\end{aligned}
$$

$$
D=0
$$

and $\operatorname{sech}(\xi)=1 / \cosh (\xi)$ denotes the hyperbolic secant. For cnoidal solutions to $\mathrm{KdV}$, one has (see [7])

$$
f(\xi)=\mathrm{cn}^{2}(\xi, m)
$$

where

$$
\begin{aligned}
& B=\sqrt{\frac{3 \alpha}{4 \beta} \frac{A}{m}}, \\
& v=1+\frac{\alpha}{2} \frac{A}{m}\left(2-m-3 \frac{E(m)}{K(m)}\right), \\
& D=-\frac{A}{m}\left(\frac{E(m)}{K(m)}+m-1\right) .
\end{aligned}
$$

Here, $\operatorname{cn}(\xi)$ is the Jacobi elliptic function, $E(m)$ is the complete elliptic integral, $K(m)$ is the complete elliptic integral of the first kind, and $m$ is the socalled elliptic parameter. The constant $D$ provides the volume conservation (the volume of the elevated part of the fluid is equal to the volume of its lowered part).

Only since 2013 (see [8]) superposition solutions are known in the form

$$
\begin{gathered}
u(x, t)=A f(\xi)+D=\frac{A}{2}\left[\operatorname{dn}^{2}(B \xi, m)\right. \\
\pm \sqrt{m} \operatorname{cn}(B \xi, m) \operatorname{dn}(B \xi, m)]+D
\end{gathered}
$$

where $\operatorname{dn}(B \xi, m)$ is also one of the Jacobi elliptic functions. The superposition solution profiles (8) are similar to the profiles of cnoidal solutions for the same $m$, but the velocity $v=1+$ $\frac{1}{8} \alpha A\left(5-m-\frac{6 E(m)}{K(m)}\right)$ is slightly different [9-11].
The KdV equation is often considered in a moving frame. For instance, applying a transformation $\hat{x}=x-t, \hat{t}=t$ to $(1)$, one obtains

$$
u_{t}+\frac{3}{2} \alpha u u_{x}+\frac{\beta}{6} u_{x x x}=0,
$$

where signs ^ are already omitted. The form of solutions (3) compared to (9) and the coefficients given by (4) $-(7)$ remain the same, only $\hat{v}=v-1$. So, the transformation to a moving frame $\hat{x}=x-t$, $\hat{t}=t$ removes the term $u_{x}$ from (1). The same term is removed when such transformation is applied to the extended $\mathrm{KdV}$, fifth-order $\mathrm{KdV}$ and Gardner equations.

The literature usually considers solutions to (1) with $A>0$. Do functions with $A<0$ also satisfy the KdV equation? Suppose that (3) satisfies (1). Therefore,

$$
A f_{t}+A f_{x}+\frac{3}{2} \alpha A^{2} f f_{x}+\frac{\beta}{6} A f_{x x x}=0 .
$$

Inversion $u \rightarrow-u$ entails $A \rightarrow-A$ and, by definition $\alpha, \alpha \rightarrow-\alpha$, but leaves $B$ and $v$ unchanged. Thus, if $u$ given by (3) with $A>0$ satisfies (1) with $\alpha>0$, then $u^{\prime}=-u$ satisfies (1) with $\alpha<0$. This is because substituting $u^{\prime}=-A f$ into $u_{t}+u_{x}+\frac{3}{2}(-\alpha) u u_{x}+\frac{1}{6} \beta u_{x x x}$ merely changes the sign of (10), i.e.,

$$
\begin{aligned}
& -A f_{t}-A f_{x}-\frac{3}{2} \alpha A^{2} f f_{x}-\frac{\beta}{6} A f_{x x x}= \\
& -\left(A f_{t}+A f_{x}+\frac{3}{2} \alpha A^{2} f f_{x}+\frac{\beta}{6} A f_{x x x}\right)=0 .
\end{aligned}
$$

The same property holds for periodic solutions, both cnoidal (4) and superposition ones (6). Note that since these periodic solutions have the form $u=A f(\xi)+D$, inversion $A \rightarrow-A$ entails $D \rightarrow-D$ and therefore $u \rightarrow-u$.

The $n$-soliton solutions are given in terms of nonlinear superpositions, see e.g. [12], where solutions with $n=2,3$ are presented in a moving frame. For $n=2$, it is still relatively easy to trace the consequences of changing the sign of the solution. Let us denote by $A_{2}>A_{1}>0$ the amplitudes of the higher and lower solitons. In the fixed frame, we introduce the notations

$$
\Theta_{i}(x, t)=\sqrt{\frac{3 \alpha}{4 \beta} A_{i}}\left[x-t\left(1+\frac{\alpha}{2} A_{i}\right)\right] .
$$

Then, 2-soliton solutions of (1) have the following form [11]

$$
\begin{aligned}
& u(x, t)=\left(A_{2}-A_{1}\right) \\
& \times \frac{A_{1} \operatorname{sech}^{2}\left(\Theta_{1}(x, t)\right)+A_{2} \operatorname{csch}^{2}\left(\Theta_{2}(x, t)\right)}{\left[\sqrt{A_{1}} \tanh \left(\Theta_{1}(x, t)\right)-\sqrt{A_{2}} \operatorname{coth}\left(\Theta_{2}(x, t)\right)\right]^{2}} .
\end{aligned}
$$

We know that the function $u(x, t)$ given by (13) for $A_{2}>A_{1}>0$ satisfies the $\mathrm{KdV}$ equation in which $\alpha>0$. Let us check how the solution (13) will look like if we change the signs of the amplitudes of the two solitons and the sign of $\alpha$, i.e., substitute $A_{i} \rightarrow-A_{i}$ and $\alpha \rightarrow-\alpha$. Since these changes do not alter the arguments of $\Theta_{i}$, we obtain 


$$
\begin{gathered}
u^{\prime}(x, t)=\frac{\left(-A_{2}+A_{1}\right)\left[-A_{1} \operatorname{sech}^{2}\left(\Theta_{1}(x, t)\right)-A_{2} \operatorname{csch}^{2}\left(\Theta_{2}(x, t)\right)\right]}{\left[\sqrt{-A_{1}} \tanh \left(\Theta_{1}(x, t)\right)-\sqrt{-A_{2}} \operatorname{coth}\left(\Theta_{2}(x, t)\right)\right]^{2}}= \\
\frac{\left(A_{2}-A_{1}\right)\left[A_{1} \operatorname{sech}^{2}\left(\Theta_{1}(x, t)\right)+A_{2} \operatorname{csch}^{2}\left(\Theta_{2}(x, t)\right)\right]}{(\mathrm{i})^{2}\left[\sqrt{A_{1}} \tanh \left(\Theta_{1}(x, t)\right)-\sqrt{A_{2}} \operatorname{coth}\left(\Theta_{2}(x, t)\right)\right]^{2}}=-u(x, t) .
\end{gathered}
$$

Indeed, the function (14) represents a 2 -soliton solution inverted with respect to (13). It satisfies (1) in which $\alpha \rightarrow-\alpha<0$. This can be verified by direct calculus, since

$$
0=-\left(u_{t}+u_{x}+\frac{3}{2} \alpha u u_{x}+\frac{\beta}{6} u_{x x x}\right)=u_{t}^{\prime}+u_{x}^{\prime}+\frac{3}{2}(-\alpha) u^{\prime} u_{x}^{\prime}+\frac{\beta}{6} u_{x x x}^{\prime} .
$$

The explicit form of 3 -soliton solution is more complicated [11, 12]. Let us assume $A_{3}>A_{2}>A_{1}>0$ and denote

$$
\begin{aligned}
& X_{1}(x, t):=-\frac{2\left(A_{1}-A_{2}\right)\left[A_{1} \operatorname{sech}^{2}\left(\Theta_{1}(x, t)\right)+A_{2} \operatorname{csch}^{2}\left(\Theta_{2}(x, t)\right)\right]}{\left[\sqrt{2 A_{1}} \tanh \left(\Theta_{1}(x, t)\right)-\sqrt{2 A_{2}} \operatorname{coth}\left(\Theta_{2}(x, t)\right)\right]^{2}} \\
& X_{2}(x, t):=\frac{2\left(-A_{1}+A_{3}\right)\left[-A_{1} \operatorname{sech}^{2}\left(\Theta_{1}(x, t)\right)+A_{3} \operatorname{sech}^{2}\left(\Theta_{3}(x, t)\right)\right]}{\left[-\sqrt{2 A_{1}} \tanh \left(\Theta_{1}(x, t)\right)+\sqrt{2 A_{3}} \tanh \left(\Theta_{3}(x, t)\right)\right]^{2}}, \\
& X_{3}(x, t):=\frac{2\left(A_{1}-A_{2}\right)}{\sqrt{2 A_{1}} \tanh \left(\Theta_{1}(x, t)\right)-\sqrt{2 A_{2}} \operatorname{coth}\left(\Theta_{2}(x, t)\right)}, \\
& X_{4}(x, t):=\frac{2\left(-A_{1}+A_{3}\right)}{-\sqrt{2 A_{1}} \tanh \left(\Theta_{1}(x, t)\right)+\sqrt{2 A_{3}} \tanh \left(\Theta_{3}(x, t)\right)} .
\end{aligned}
$$

Then 3-soliton solution is expressed with (16)-(19) as

$$
\begin{aligned}
& u(x, t)=A_{1} \operatorname{sech}^{2}\left[\Theta_{1}(x, t)\right] \\
& -2\left(A_{2}-A_{3}\right) \frac{X_{1}(x, t)+X_{2}(x, t)}{\left(X_{3}(x, t)-X_{4}(x, t)\right)^{2}} .
\end{aligned}
$$

With the same arguments, we see that the inversion $A_{i} \rightarrow-A_{i}$, where $i=1,2,3$, implies $u^{\prime} \rightarrow-u$ in $(20)$. Therefore, the inverted 3 -soliton solution fulfilles the $\mathrm{KdV}$ equation with $\alpha^{\prime}=\alpha$.

Analogously, similar arguments hold for $n$-soliton solutions and for $\mathrm{KdV}$ equation in a moving frame (9).

\subsection{Extended KdV equation (KdV2)}

The extended KdV equation (KdV2), first derived by Marchant and Smyth [13], has in the fixed reference frame the following form

$$
\begin{aligned}
& u_{t}+u_{x}+\frac{3}{2} \alpha u u_{x}+\frac{\beta}{6} u_{x x x}-\frac{3}{8} \alpha^{2} u^{2} u_{x} \\
& \quad+\alpha \beta\left(\frac{23}{24} u_{x} u_{x x}+\frac{5}{12} u u_{x x x}\right)+\frac{19 \beta^{2}}{360} u_{x x x x x}=0 .
\end{aligned}
$$

Both equations, KdV (1) and KdV2 (21), are derived from the model of an ideal fluid (incompressible and inviscid) with an irrotational motion under the assumption that $\alpha=\mathcal{O}(\beta)$, that is, the parameters $\alpha, \beta$ are small and of the same order. Applying the perturbation technique, one obtains the $\mathrm{KdV}$ equation when the approach is limited to the first order in small parameters and the extended $\mathrm{KdV}$ equation $(\mathrm{KdV} 2)$ when the derivations are extended to the second order.

Contrary to the $\mathrm{KdV}$ equation, (21) is nonintegrable. Despite this fact, it has analytic singlesoliton solutions [14], as well as periodic cnoidal solutions [7] and superposition solutions [9, 10]. However, the multi-soliton solutions to (21) do not exist [15].

The analytic single-soliton and periodic solutions of KdV2 have the same functional form as the analogous solutions of $\mathrm{KdV}$ but the corresponding coefficients $B, v$ are slightly different, and the coefficient $A$ is determined by the parameters of the equation, i.e., by $\alpha, \beta$. It is because $\mathrm{KdV} 2 \mathrm{im}-$ poses one more condition on the coefficients $A$, $B, v$ determining the solution, than $\mathrm{KdV}[7,9$ $11,14]$. For $\mathrm{KdV}$, the set of the coefficients has one degree of freedom, which, for fixed $\alpha, \beta$, allows the existence of solitons of different heights, and thus allows for multi-soliton solutions. For KdV2 with fixed $\alpha, \beta$, there exists a soliton with only one possible height, so multi-soliton solutions do not exist [15].

If the function $u(x, t)$ satisfies $\mathrm{KdV} 2$, it is easy to see that the inverted function $u^{\prime}=-u$ satisfies (21) with the sign of $\alpha$ changed $\alpha^{\prime}=-\alpha$ because

$$
\begin{aligned}
& u_{t}^{\prime}+u_{x}^{\prime}+\frac{3}{2} \alpha^{\prime} u^{\prime} u_{x}^{\prime}+\frac{\beta}{6} u_{x x x}^{\prime}-\frac{3}{8} \alpha^{\prime 2} u^{\prime 2} u_{x}^{\prime} \\
& +\alpha^{\prime} \beta\left(\frac{23}{24} u_{x}^{\prime} u_{x x}^{\prime}+\frac{5}{12} u^{\prime} u_{x x x}^{\prime}\right)+\frac{19 \beta^{2}}{360} u_{x x x x x}^{\prime}= \\
& -u_{t}-u_{x}-\frac{3}{2} \alpha u u_{x}-\frac{\beta}{6} u_{x x x}+\frac{3}{8} \alpha^{2} u^{2} u_{x} \\
& -\alpha \beta\left(\frac{23}{24} u_{x} u_{x x}+\frac{5}{12} u u_{x x x}\right)-\frac{19 \beta^{2}}{360} u_{x x x x x}= \\
& -\left(u_{t}+u_{x}+\frac{3}{2} \alpha u u_{x}+\frac{\beta}{6} u_{x x x}-\frac{3}{8} \alpha^{2} u^{2} u_{x}\right. \\
& \left.+\alpha \beta\left(\frac{23}{24} u_{x} u_{x x}+\frac{5}{12} u u_{x x x}\right)+\frac{19 \beta^{2}}{360} u_{x x x x x}\right)=0 .
\end{aligned}
$$

This conclusion remains valid also in a moving frame of reference. 


\subsection{Fifth-order KdV equation}

Note that $\mathrm{KdV}$ and KdV2 were derived completely neglecting the pressure exerted by the surface tension of the deformed liquid surface. The terms derived from the surface tension include a coefficient called the Bond number $\tau=T /\left(\varrho g H^{2}\right)>0$, where $T$ is the surface tension coefficient of the liquid, $\varrho$ is its density, $g$ is the standard acceleration of free fall and $H$ is the depth of the reservoir. For water, at a depth of $H \geq 1 \mathrm{~m}$, we have $T \leq 10^{-7}$, so the effect of surface tension can be completely neglected. For thin liquid layers, in turn, when $H$ is on the order of millimeters, the terms derived from the surface tension become significant [16].

Taking into account the terms originating from surface tension in the Euler equations, assuming $\alpha=\mathcal{O}\left(\beta^{2}\right)$, and applying perturbation approach up to second order in small parameters, one derives so-called fifth-order KdV equation (see [17, 18])

$$
\begin{gathered}
u_{t}+u_{x}+\frac{3}{2} \alpha u u_{x}+\frac{(1-3 \tau) \beta}{6} u_{x x x} \\
+\frac{\left(19-30 \tau-45 \tau^{2}\right) \beta^{2}}{360} u_{x x x x x}=0 .
\end{gathered}
$$

It is well known, see, e.g. [19, 20], that the fifthorder $\mathrm{KdV}$ equation has a form of soliton solution

$$
u(x, t)=A \operatorname{sech}^{4}[B(x-v t)] .
$$

Here it is also easy to see that if $u$ satisfies (23) then the function $u^{\prime}=-u$ satisfies (23) with $\alpha^{\prime}=-\alpha$.

\subsection{Gardner equation}

Assuming $\beta=\mathcal{O}\left(\alpha^{2}\right)$, the so-called Gardner equation [18] is derived from Euler's equations with second order accuracy in small parameters. It is given as

$u_{t}+u_{x}+\frac{3}{2} \alpha u u_{x}-\frac{3}{8} \alpha^{2} u^{2} u_{x}+\frac{(1-3 \tau) \beta}{6} u_{x x x}=0$.

It is a well-known fact that for the Gardner equation (25) there exists one-parameter family of analytic solutions in the form $[5,21]$

$$
u(x, t)=\frac{A}{1+B \cosh \left[\frac{1}{\Delta}(x-v t)\right]} .
$$

The functions (26) also represent solitons with fixed profiles, moving at a constant speed. Their shapes can be very different, from bell-shaped solitons to table-top ones, depending on values of equation parameters [5, 21].

Since three conditions are imposed on the coefficients $A, B, v, \Delta$ in (25), so three of these coefficients can be expressed as functions of one. Choosing $\Delta$ as an independent parameter, one obtains the relations (denoting $(1-3 \tau) \beta / 6=\beta^{\prime}$ )

$$
\begin{aligned}
& A=\frac{4 \beta^{\prime}}{\alpha \Delta^{2}}, \quad B= \pm \sqrt{1-\frac{\beta^{\prime}}{\Delta^{2}}}, \\
& v=1+\frac{\beta^{\prime}}{\Delta^{2}} .
\end{aligned}
$$

As the parameters $B$ and $v$ do not depend on $\alpha$, so the inverted function $u^{\prime}=-u$ requires $\alpha^{\prime}=-\alpha$ with $B, v, \Delta$ unchanged. In this case, it can also be seen that if $u$ satisfies (25), then $u^{\prime}=-u$ also satisfies (25) with $\alpha^{\prime}=-\alpha$.

\section{Uneven bottom}

Back in 2014, in the work [14], we started the systematic study on the extension of the $\mathrm{KdV}$ and $\mathrm{KdV} 2$ equations to the more general case in which the bottom of the tank is described by a continuous bounded function $h(x)$. To this end, we have introduced a new small parameter $\delta=\frac{a_{h}}{H}$, where $a_{h}$ denotes the amplitude of the bottom profile.

However, it is only in [16] that we have fully realized this goal. In fact, we have shown that the new term in these nonlinear wave equations is universal, i.e., it is the same for all four equations: $\mathrm{KdV}, \mathrm{KdV} 2$, fifth-order $\mathrm{KdV}$, and Gardner. The same constraint holds in all these cases. Namely, the system of Boussinesq equations can be reduced to a single wave equation only if the bottom function satisfies the condition $h_{x x}=0(h(x)$ is a piecewise linear function). In such cases, the generalized $\mathrm{KdV}$, $\mathrm{KdV} 2$, fifth-order $\mathrm{KdV}$ and Gardner equations take the form

$$
E Q-\frac{1}{4} \delta\left(2 h u_{x}+h_{x} u\right)=0
$$

where $E Q$ stands for left hand side of (1), (21), (23) or $(25)$.

Note that (28) is non-integrable. Based on the analysis in Sect. 2, we know that if $u$ satisfies the equation $E Q=0$, then $u^{\prime}=-u$ satisfies $E Q^{\prime}=0$ in which $\alpha^{\prime}=-\alpha$, because then every term of the equation will change sign. It can be seen that changing $u \rightarrow-u$ will also change the sign of the entire term $-\frac{1}{4} \delta\left(2 h u_{x}+h_{x} u\right)$, if $\delta$ remains unchanged. Thus, if the function $u(x, t)$ is a solution to one of the generalized equations (28) (i.e., the $\mathrm{KdV}, \mathrm{KdV} 2$, 5 th-order $\mathrm{KdV}$, or Gardner equations), then the inverted function $-u(x, t)$ is the solution of the corresponding one of these equations, in which $\alpha$ is replaced by $-\alpha$.

\section{Conclusions}

The inverted solutions of the Korteveg-de Vries $(\mathrm{KdV})$, extended $\mathrm{KdV}(\mathrm{KdV} 2)$, fifth-order $\mathrm{KdV}$, and Gardner equations satisfy these equations with a negative nonlinearity parameter $\alpha$. The same property holds for the $\mathrm{KdV}$, extended $\mathrm{KdV}$, fifthorder $\mathrm{KdV}$, and Gardner equations generalized for uneven bottom in [16].

Solutions of nonlinear wave equations of the $\mathrm{KdV}$ and Gardner type with negative amplitudes can have significant physical applications. The analysis of giant depression-type internal waves that can form in deep seas at the boundary of areas with different water densities and salinity carried out in [6], indicates that these phenomena can be the cause of submarine disasters. 


\section{References}

[1] J.S. Russel, Report on Waves in: Fourteenth meeting of the British Association for the Advancement of Science, Report 311, 1844.

[2] J.V. Boussinesq, "Théorie générale des mouvements qui sont propagés dans un canal rectangulaire horizontal." C.R. Acad. Sci. 73256 (1871).

[3] D.J. Korteweg, H. de Vries, Philos. Mag. 39422 (1895).

[4] E. Falcon, C. Laroche, S. Fauve, Phys. Rev. Lett. 89204501 (2002).

[5] L. Ostrovsky, E Pelinovsky, V. Shrira, Y. Stepanyants, Chaos 25097620 (2015).

[6] Y. Stepanyants, arXiv:2107.00828, 2021.

[7] E. Infeld, A. Karczewska, G. Rowlands, P. Rozmej, Acta Phys Pol. A 1331191 (2018).

[8] A Khare, A. Saxena, J. Math. Phys. 55 032701 (2013).

[9] P. Rozmej, A. Karczewska, Adv. Math. Phys. 20185095482 (2018).

[10] P. Rozmej, A. Karczewska, E. Infeld, Nonlinear Dyn. 911085 (2018).

[11] A. Karczewska, P. Rozmej, Shallow water waves - extended Korteweg-de Vries equations, Oficyna Wydawnicza Uniwersytetu Zielonogórskiego, Zielona Góra 2018.
[12] K. Brauer, "The Korteweg-de Vries Equation: History, Exact Solutions, and Graphical Representation", University of Osnabrück, 2000.

[13] T.R. Marchant, N.F. Smyth, J. Fluid Mech. 221263 (1990).

[14] A. Karczewska, P. Rozmej, E. Infeld, Phys. Rev. E 90012907 (2014).

[15] A. Karczewska, P. Rozmej, Acta Phys. Pol. A 136910 (2019).

[16] A. Karczewska, P. Rozmej, Commun. Nonl. Sci. Numer. Simulat. 82105073 (2020).

[17] J.K. Hunter, J. Scheurle, Physica D 32253 (1988).

[18] G.I. Burde, A. Sergyeyev, J. Phys. A Math. Theor. 46075501 (2013).

[19] B. Dey, A. Khare, C.N. Kumar, Phys. Lett. A 223449 (1996).

[20] T.J. Bridges, G. Derks, G. Gottwald, Physica D 172190 (2001).

[21] R. Grimshaw, E. Pelinovsky, T. Talipova, Physica D 13240 (1999). 